\title{
Dynkin diagram strategy for orbifolding with Wilson lines
}

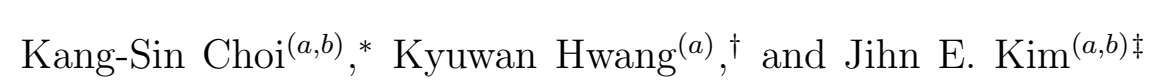 \\ ${ }^{(a)}$ School of Physics and Center for Theoretical Physics, \\ Seoul National University, Seoul 151-747, Korea, and \\ (b) Physikalisches Institut, Universität Bonn, \\ Nussallee 12, D53115, Bonn, Germany
}

\begin{abstract}
A simple method for breaking gauge groups by orbifolding is presented. We extend the method of Kac and Peterson to include Wilson lines. The complete classification of the gauge group breaking, e.g. from heterotic string, is now possible. From this Dynkin diagram technique, one can easily visualize the origin and the symmetry pattern of the surviving gauge group.
\end{abstract}

PACS numbers: 02.20.Qs, 11.25.Mj, 11.30.Ly

Keywords: orbifold compactification, Dynkin diagram, Wilson lines

*Electronic address: ugha@phya.snu.ac.kr

$\dagger$ Electronic address: kwhwang@phya.snu.ac.kr

${ }^{\ddagger}$ Electronic address: jekim@phyp.snu.ac.kr 


\section{INTRODUCTION}

The heterotic string introduced an interesting simply-laced semi-simple group $E_{8} \times E_{8}^{\prime}$ in 10 dimension(10D) which can be a candidate for the fundamental theory near the Planck scale 1]. However, one must compactify the six internal spaces so as to reconcile with the observed 4 dimensional(4D) physics. Among a few directions for compactification, the orbifolding has been known to be especially simple and efficient, and gives a rich spectra for 4D particles [2, 3]. In addition, this orbifold method has led to interesting 4D models which exhibit some desirable physical phenomena such as the standard model gauge group and the doublet-triplet splitting[4].

The so-called standard-like models, leading to the gauge group $S U(3) \times S U(2) \times U(1)^{n}$ with three chiral families, have been constructed vigorously along this line 4, 5, 6]. In case of no Wilson line, there even exist extensive tables for all $Z_{N}$ orbifolds[7]. However, the possibilities for inclusion of Wilson lines are exponentially larger than those without Wilson lines. Therefore, the inclusion of Wilson line(s) was limited to the study toward the standard-like models. Along this line, an equivalence relation was even devised to ease the study [8].

However, this initial study toward string derivation of the supersymmetric standard model could not overcome two serious hurdles along this direction, one the $\sin ^{2} \theta_{W}$ problem and the other the problem of too many Higgs doublets. Therefore, recently the orbifold has been tried at the field theory level[9]. Here, the doublet-triplet splitting problem [9] and the flavor problem [10] have been reconsidered, but it did not exhibit a predictive power due to the arbitrariness of the field content introduced at the fixed points.

This led us to consider the string orbifolds again. One recent suggestion has been that a semi-simple gauge group such as $S U(3)^{3}$ is plausible for a unification group at high energy to solve the $\sin ^{2} \theta_{W}$ problem in the orbifold compactification[11]. This opens a new door toward string orbifolds. In this respect, it is worthwhile to consider possible string vacua through orbifold compactifications. Of course, the previous classifications can be useful, but they are not complete in that there does not exist a complete classification with Wilson lines. Therefore, in this paper we try to investigate how to implement the Wilson lines without much computer time.

In this study, the simple group theoretical method, originally devised by Kac and Peter- 
son [12], is found to be extremely useful. This method uses the Dynkin diagram, and can be understood pictorially. In this method, we can easily tell the origin and the symmetry of the resulting gauge group. However, cases with more than one shift vector, which is the case of our interest with Wilson lines, have not been studied completely. Here, generalizing Kac and Peterson, we present a systematic search criteria for the gauge groups in cases with

more than one shift vector. And we seek the behind symmetry of group, which is in fact apparent in the Dynkin diagram. With the criteria present in this paper, it is in principle possible to classify the groups completely.

\section{ORBIFOLD AND SHIFT VECTOR}

\section{A. Breaking the group by a shift vector}

We begin with the conventional root space the dimension of which is the rank of the group. Let us restrict the discussion to the self dual lattices. For a group $G$ and its root lattice spanned by its roots $P$, we can make a transformation of $P$ by the shift vector $V$,

$$
\begin{aligned}
& |P\rangle \mapsto e^{2 \pi i P \cdot V}|P\rangle, \\
& |Q\rangle \mapsto|Q\rangle,
\end{aligned}
$$

where we have casted roots as states and $|Q\rangle$ is the set of Cartan generators. If we require that this transformation is the symmetry of the system, it breaks the group $G$ into its subgroup $H$, which consists of the root vectors $|P\rangle$ satisfying

$$
P \cdot V=\text { integer }
$$

The order of the shift vector $V$ is defined to be the minimum integer number $N$ such that $N$ successive transformation becomes the identity operation up to a lattice translation, i.e., $N V$ belongs to the root lattice.

\section{B. Orbifold}

The orbifold embedding of shift vector is a natural realization of this property. An orbifold is defined by moding out the manifold $\mathbf{R}^{n}$ by the space group $\mathcal{S}$, which is seen equivalently 
as moding out the torus $T^{n}$ by the point group $\mathcal{P}$,

$$
\mathbf{R}^{n} / \mathcal{S}=T^{n} / \mathcal{P}
$$

Under $\mathcal{S}$, an element $x$ of $\mathbf{R}^{n}$ transforms as,

$$
x \mapsto \theta x+v .
$$

Here, the (usually rotation) element $\theta$ also belongs to the point group $\mathcal{P}$, or the automorphism of the lattice vector defining the torus. The order $N$ has the same meaning as that of the shift vector, i. e., $\theta^{N}=1$. Naively speaking, the orbifolding is the identification of points on $T^{n}$ up to the transformation $\theta$. We associate this space group transformation with the gauge group transformation,

$$
\begin{gathered}
\text { "rotation" by } \theta \rightarrow e^{2 \pi i P \cdot V} \\
\text { translation by } v \rightarrow e^{2 \pi i P \cdot a} \text {. }
\end{gathered}
$$

We refer the latter as the Wilson line shift vector.

\section{Symmetries of Lie group}

A simple Lie group has many symmetries. Any set of shift vectors which are connected by these symmetries are equivalent, leading to the same subgroup.

1. Lattice translation: Although this is not a symmetry of the gauge group, it is the redundancy of the formulation by the shift vector. Under the translation

$$
V \mapsto V+\alpha
$$

by a root vector $\alpha$, the condition (3) does not change, since the root vector is also an element of the dual lattice (self dual), any vector of which has only integer value when taking the dot product with any root vector.

2. Weyl reflection: The Weyl reflection $\left(\sigma_{\alpha}\right)$ is defined as a reflection about the plane whose normal vector is the $\operatorname{root}(\alpha)$ of the group.

$$
V \mapsto \sigma_{\alpha} V=\sigma_{-\alpha} V=V-2 \frac{\alpha \cdot V}{\alpha^{2}} \alpha=V-(\alpha \cdot V) \alpha
$$

We know that the set of any number of successive reflections form a group. The group generated by the Weyl reflections is called Weyl group. 
3. Outer automorphism: The Dynkin diagram, which is a diagrammatical representation of the Cartan matrix, contains almost every information of the given group. Every small circle(or bullet) represents a simple root and the linking lines rerepresents the angle between the simple roots linked.[In our case, we consider only small circles and singly connected lines.] Some Dynkin diagram possesses some exchange symmetry, which is the outer automorphism of the group. Some of the previous works classifying orbifold models neglected this possibility. In fact, many breaking patterns turn out to be the identical one.

\section{DYNKIN DIAGRAM TECHNIQUE FOR SEACHING SURVIVING GROUPS}

What will be the subgroup $H$ which survives the condition Eq.(3) ? The basic method of finding the group structure is to identify the simple roots for the set of all roots which survive the projection (3). By choosing the form of the shift vector carefully, one can find the simple roots without identifying all roots surviving the projection. For the case of one

shift vector, i.e. when there is no wilson line shift, Kac and Peterson [12] introduced a very useful choice of such form of the shift vector.

Let us concentrate on the group $E_{8} . E_{8}$ has eight simple roots $\alpha^{i}, i=1 . .8$, represented by the small circles in the (extended) Dynkin diagram in FIG.(11). The highest root $\theta$ of $E_{8}$ is given by

$$
\theta=2 \alpha^{1}+3 \alpha^{2}+4 \alpha^{3}+5 \alpha^{4}+6 \alpha^{5}+4 \alpha^{6}+2 \alpha^{7}+3 \alpha^{8} .
$$

Let us define the Coexter label $\left\{n_{i}\right\}$ as the coefficients of $\alpha^{i}$ in this simple root expansion :

$$
\theta=\sum_{i=1}^{r} n_{i} \alpha^{i}
$$

where $r=\operatorname{rank}$ of $G$. If we define $\alpha^{0}=-\theta$, its dot product with the other simple roots vanishes except with $\alpha^{1}$, for which it is -1 . It cannot be included in the set of simple roots of $E_{8}$, since $\alpha^{0}$ is not linearly independent on the other simple roots $\alpha^{i}$ nor a positive root. However, it can be a candidate of new simple root when some simple root fails to pass the criterion Eq.(3). This set is called an extended root system $\widehat{G}$ and the extended Dynkin diagram is shown in FIG.(11).

Let us expand the shift vector in the basis of the fundamental weight, namely Dynkin 


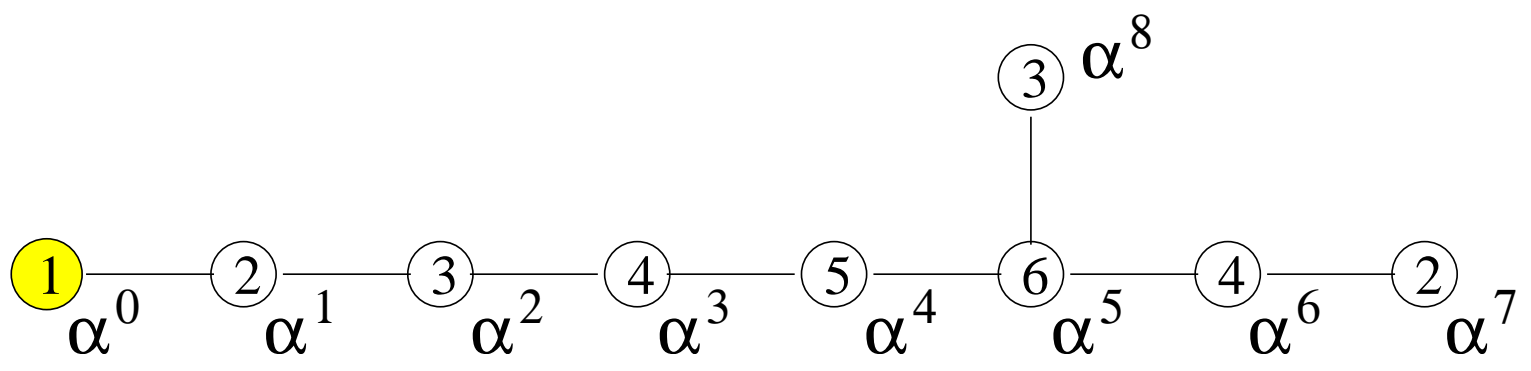

FIG. 1: Extended Dynkin diagram of $\widehat{E_{8}}$ group. The numbers in the circle are the Coexter label $n_{i}$ of the corresponding simple roots.

basis $\left\{\gamma_{i}\right\}$, satisfying $\gamma_{i} \cdot \alpha^{j}=\delta_{i}^{j}$,

$$
V=\frac{1}{N} \sum_{i=1}^{r} s_{i} \gamma_{i}
$$

where $N$ is the order of the shift vector and $s_{i}$ is given by $N \alpha^{i} \cdot V$. We can limit our consideration for the shift vector satifying

$$
\sum_{i=1}^{8} n_{i} s_{i} \leq N, \quad s_{i} \geq 0,
$$

for it is well known that one can always transform the shift vector into this 'standard form' by lattice translation and Weyl reflection of $E_{8}$.

With this form of shift vector, it is easy to identify the simple roots of the surviving roots. Let us define the set of indices $J$ which is made of the indices $i$ for which $s_{i}$ does not vanish.

$$
J=\left\{i \mid s_{i} \neq 0\right\}
$$

First, let us consider the case where the inequality of Eq.(8) holds. For any positive root $P$ of $E_{8}$

$$
P=\sum_{i=1}^{8} c_{i} \alpha^{i}, \quad c_{i} \geq 0,
$$

the projection condition Eq.(3) becomes

$$
P \cdot V=\sum_{i \in J} c_{i} s_{i}=\text { integer } .
$$

This value is always smaller than one since that for the highest root is less than one.

$$
P \cdot V \leq \theta \cdot V=\sum_{i \in J} n_{i} \frac{s_{i}}{N}<1
$$


Thus, any root having non-zero $c_{i}$ for $i \in J$, fails to pass the condition. This means we can safely remove the corresponding circle in the Dynkin diagram without causing any side effect. The resulting subgroup can be read off from the resulting diagram.

Now, let us consider the case when the equality in Eq.(8) holds. In this case, there are some roots, which still survives the projection condition even though $c_{i} \neq 0$ for some $i \in J$, which means we cannot just remove the corresponding circle in the Dynkin diagram. Let us call the set of such roots as $A$. Any positive root $\tilde{P}$ in $A$ has $c_{i}=n_{i}$ for all indices $i \in J$, hence it is expressed as

$$
\tilde{P}=\theta-\sum_{i \notin J} b_{i} \alpha^{i}=-\alpha^{0}-\sum_{i \notin J} b_{i} \alpha^{i}, \quad b_{i} \geq 0,
$$

for some non-negative integer $b_{i}$. Hence any negative root $-\tilde{P}$, being the minus of $\tilde{P}$, in $A$ can be expressed by the sum of the root vectors $\left\{\alpha^{0}, \alpha^{i}\right.$ for $\left.i \notin J\right\}$ with positive coefficients. Thus, by changing the definition of positive root for the root in $A$ in such a way that a previous negative root is a positive root and vise versa, one can confirm that $\left\{\alpha^{0}, \alpha^{i}\right.$ for $i \notin J\}$ forms a proper set of simple roots. Thus the surviving subgroup is represented by the extended Dynkin diagram with $i$-th circle being removed for $i \in J$, i.e. $s_{i} \neq 0$.

Observing that whether we add the extended simple root $\alpha^{0}$ to the Dynkin diagram or not depends on the value $s_{0}$ defined by

$$
s_{0} \equiv N(1-\theta \cdot V)=N-\sum_{i=1}^{8} n_{i} s_{i}
$$

vanishes or not, both cases considered in the last two paragraphs can be treated universally by formaly defining the Coexter label $n_{0}$ for the extended simple root $\alpha^{0}$ as 1 and restrict our consideration to the case which satisfies

$$
\sum_{I=0}^{8} n_{I} s_{I}=N, \quad s_{I} \geq 0 .
$$

For a solution $\left\{s_{I}, I=0 . .8\right\}$ of Eq.(15), the unbroken subgroup by the shift vector given by Eq.(17) can be read off from the extended Dynkin diagram with $I$-th circle removed for any index $I$ with $s_{I} \neq 0$ including $I=0$. If the rank of the diagram is smaller than 8 , there are additional $U(1)$ 's which fill up the rank to 8. The trivial case is $s_{0}=N$ where the group $G$ remains unbroken.

It will be interesting to think about what will be the unbroken subgroup for the shift vector which does not satisfy the condition Eq.(8). For example, take $V=\frac{1}{3} \gamma_{5}$, hence 
$\sum n_{i} s_{i}=6>N$ where $N=3$. It is tempting to speculate that deleting the 5 th $\operatorname{circle}\left(\alpha_{5}\right)$ in the extended Dynkin diagram resulting in $S U(6) \times S U(2) \times S U(3)$. However, the set $\left\{\alpha^{0}, \alpha^{i}\right\}-\left\{\alpha^{5}\right\}$ cannot generate a root vector with $c_{5}=3$ which survives the projection. In this case, the proper simple root turns out to be

$$
\alpha=\alpha^{3}+2 \alpha^{4}+3 \alpha^{5}+2 \alpha^{6}+\alpha^{7}+\alpha^{8} .
$$

It links to $\alpha^{2}$ and $\alpha^{8}$, whose resulting "extended" Dynkin diagram is again $E_{6} \times S U(3)$, which is depicted in FIG. 2. By the theorem above, there is an equivalent shift vector obeying the condition (8) leading to the same subgroup. One can show that this is an equivalent breaking to the second one in TABLE [, by lattice translations and Weyl reflections.

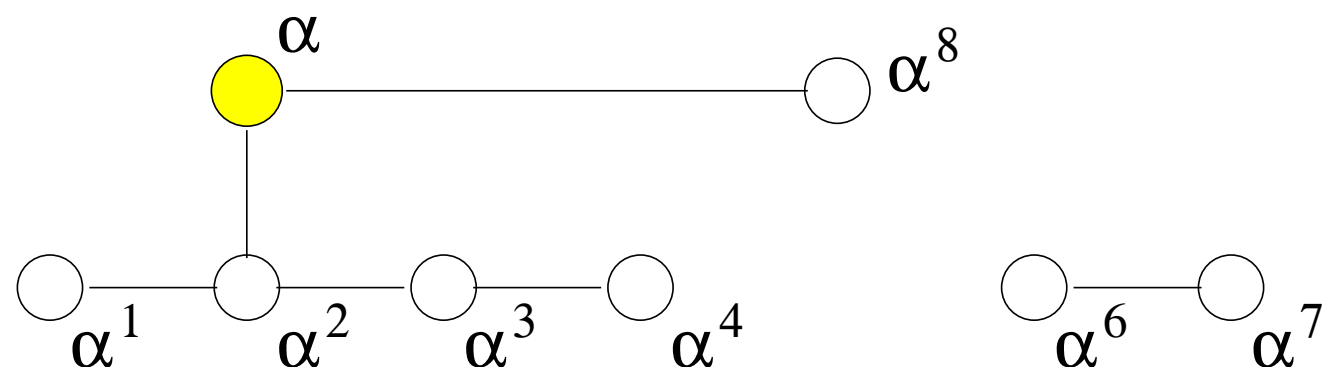

FIG. 2: An order $N=3$ breaking. A shift vector which deletes the $5^{\text {th }}$ spot leads to $E_{6} \times S U(3)$. Compare this with FIG. 1.

The complication of this example comes from the fact that there are surviving roots which cannot be expressed in terms of the highest root and the surviving simple roots. Those roots are allowed since $\theta \cdot V=\sum n_{i} s_{i} / N$ is greater than 1 . Hence this example shows the importance of the condition Eq.(88) in reading off the gauge group directly from the Dynkin diagram.

The power of the condition Eq. (15) lies not only in the easyness to identify the unbroken subgroup but also in the fact that there are only a few possible set of $s_{I}$ 's that can satisfy it. Thus the procedure of finding all possible gauge symmetry breaking by the shifts can be very simplified using the condition Eq.(15) and the extended Dynkin diagram. For example, let us consider an order $N=3$ shift. From Eq. (15) and watching $n_{I}$, there are only five possibilities. The shift vector (77) in the Dynkin basis and the corresponding five unbroken groups are listed in TABLE \. 


\begin{tabular}{cc}
\hline$\left[s_{i} \mid s_{0}\right]$ & unbroken group $H$ \\
\hline$[00000000 \mid 3]$ & $E_{8}$ \\
{$[01000000 \mid 0]$} & $E_{6} \times S U(3)$ \\
{$[00000001 \mid 0]$} & $S U(9)$ \\
{$[10000000 \mid 1]$} & $E_{7} \times U(1)$ \\
{$[00000010 \mid 1]$} & $S O(14) \times U(1)$ \\
\hline
\end{tabular}

TABLE I: The order $N=3$ breakings of $E_{8}$. The last entry $s_{0}$ can be added automatically to satisfy Eq. (15).

\section{INTRODUCTION OF WILSON LINE}

In addition to the shift vector associated with the point group, we introduce another shift vector when we turn on a Wilson line [3] $a$, which should also satisfy the same condition as $V$ and is required to satisfy additional conditions on the modular invariance stated in the next section. Extending the diagramatic method of finding the unbroken subgroup for the first breaking of $E_{8}$, we can find a well-defined procedure of finding the unbroken subgroup for the further breaking by the additional Wilson line shift vector. One can introduce as many Wilson lines as the number of the compact dimensions in the orbifold compactification bases on torus. Most of the statements in this section assumes that the procedure is applied recursively for each additional Wilson line $a_{i}$. Some of the statements is for the first Wilson line $a_{1}$, for the sake of definiteness and simpleness of the argument, though. These statements can be generalized appropriately to the next Wilson line easily.

Let us call the unbroken subgroup of the first breaking in the previous section as $H=$ $H_{1} \times H_{2} \times \ldots$, where each $H_{x}$ is a simple group. The first step of the method in the previous section was to find an efficient form of a shift vector expanded in the dual basis, i.e. the fundamental weight of $E_{8}$. For now we are dealing with the subgroup of $E_{8}$, one might try to expand in terms of the fundamental weights of $H_{x}$. However, it is not useful since the fundamental weight vectors of $H_{x}$ are not on the root lattice of $H_{x}$ since it does not form a self-dual lattice in general. For some cases, they reside in the root lattice of $H_{x}$ if they are multiplied by some positive integer $\hat{N}$, implying they might be useful for $Z_{\hat{N}}$ orbifold. Still, it lacks the generalities and do not allow the formulation for the general order $N$, hence we 
do not use them in this paper. Instead, we keep using the expansion by the fundamental weights of $E_{8}, \gamma_{i}$. They are not exactly a dual of the simple roots we found in the previous section in the strict sence, since $\alpha^{0}$, the negative of the highest root of $E_{8}$, which does not have its dual among $\gamma_{i}$, can be one of the simple roots of $H$. However, this expansion is quite useful since all the other simple roots of $H$ are still identified as simple roots of $E_{8}$. For the simple root $\alpha^{0}$, if present, whether the corresponding circle should be removed or not can be deduced from the coefficients of the other simple roots. The central point of our method is to provide a neat formulation of keeping track of the fate of those extended simple roots at the previous stage in a similar form of Eq.(15).

We start our discussion by defining the highest root $\theta^{x}=-\alpha^{H_{x}}$ and the labels $\left\{n_{I}^{H_{x}}\right\}$ for each simple group factor $H_{x}$ as

$$
\theta^{x}=\sum_{i \in J_{x}} n_{i}^{H_{x}} \alpha^{i}
$$

where $J_{x}$ is the set of indices $i$ for which the $E_{8}$ simple root $\alpha^{i}$, including $\alpha^{0}$, is identified to be a simple root of $H_{x}$ at the previous stage of breaking. By adding $\alpha^{H_{x}}$, the root system of each $H_{x}$ is extended to $\widehat{H_{x}}$. We expand the Wilson line shift vector $a$ in terms of the fundamental weights of $E_{8}$.

$$
a=\frac{1}{N} \sum_{i=1}^{8} w_{i} \gamma_{i}
$$

The next important step is to reduce the possibilities of $w_{i}$ by requiring the analogous condition of Eq.(8). For each simple group $H_{x}$, we can transform a shift vector $a$ into its own standard form by the lattice translation and the Weyl reflection by the root of $H_{x}$. Here we can not use the standard form itself since we do not expand $a$ in terms of the fundamental weight of $H$. Still, a slightly different form of the same theorem is very useful to constrain the shift vector $a$ :

Theorem : One can always transform any vector $a$ in the combined root space of $H=$ $H_{1} \times H_{2} \times \ldots$ by lattice translation and Weyl reflection by the root of $H_{x}$ into the one that satisfies

$$
N \theta^{x} \cdot a=\sum_{i \in J_{x}} n_{i}^{H_{x}}\left(N \alpha^{i} \cdot a\right) \leq N, \quad \alpha^{i} \cdot a \geq 0
$$

for each simple group $H_{x}$. This transformation does not change the set of shift vectors $\left\{V, a_{1}, \ldots\right\}$ which leads to the symmetry breaking $E_{8} \rightarrow H$, upto lattice translation. 
The proof of the first statement of the theorem is manifest since it is just the substitution of $s_{i}$ by $N \alpha^{i} \cdot a$ in Eq.(8) and each simple group $H_{x}$ is completely independent among one another since $\alpha^{i} \cdot \alpha^{j}=0$ if $\alpha^{i}$ and $\alpha^{j}$ belong to different subgroup. The second statement of the theorem, which is an important consistency condition of our scheme, is also obvious since any root $P$ which survive the projection of the previous stage satisfies $P \cdot V=$ integer, $P \cdot a_{1}=$ integer, etc.

For the sake of simplicity, we illustrate the usage of this theorem in dealing with the extended root $\alpha^{0}$ of $E_{8}$ only. The generalization to the other extended roots for the subgroup of $E_{8}$ can be made easily. For the simple group $H_{x}$ which does not contain $\alpha^{0}$ as its simple root, this theorem is simply equivalent to

$$
\sum_{i \in J_{x}} n_{i}^{H_{x}} w_{i} \leq N, \quad w_{i} \geq 0
$$

just like the $E_{8}$ case in the first breaking by $V$. For the simple group $H_{x}$ which contains $\alpha^{0}$, it translates into

$$
\begin{aligned}
N \theta^{x} \cdot a & =\sum_{i \in J_{x}-\{0\}} n_{i}^{H_{x}} w_{i}+n_{0}^{H_{x}} w_{0} \leq N, \quad w_{i}, w_{0} \geq 0, \\
w_{0} & \equiv N \alpha^{0} \cdot a=-\sum_{i=1}^{8} n_{i} w_{i} .
\end{aligned}
$$

The non-trivial condition on the quantity $w_{0} \geq 0$ is the simple consequance of the theorem. The apparent sign inconsistency between Eq.(21) and Eq.(19),(20) is to be resolved by the negative value of $w_{i}$ with $i \in J$, where $J$ is the set of all indices $i$ for which $\alpha^{i}$ fails to pass the projection at the previous stage. Thus $w_{i}$ for $i \in J$ should be fixed by hand in such a way that $w_{0}$ defined by Eq.(21) satisfies Eq.(20). When $J$ has only one element $\hat{i}$, the constraints on $w_{0}$ is equivalent to find a solution of

$$
\sum_{I=0}^{8} n_{i} w_{i}=N \quad \bmod N
$$

with $w_{\hat{\imath}}=0$ since $n_{\hat{i}}$ is already a multiple of $N$. This property is useful for some physically interesting cases like further breaking of $S U(3) \times E_{6}$ on $Z_{3}$ orbifold which we will see in detail in the next section. When $J$ has more than one element, there may be more than one way for $w_{i}, i \in J$ to satisfy Eq.(21). Each such solution may or may not lead to the same gauge group, depending on whether they are related to one another by Weyl reflection or not. It 
cannot be knwon at this stage in general way. Thus, for the classification purpose, they must be treated as a different one unless they are proven to be related by Weyl reflection.

Given the restriction of Eq.(19),(20), with the proper definition of $w_{0}$ in Eq.(21), we can define the coefficient $w_{0}^{H_{x}}$ for the extended root $\alpha^{H_{x}}$ of the simple group factor $H_{x}$ such that

$$
\sum_{I \in J_{x}} n_{i}^{H_{x}} w_{i}+w_{0}^{H_{x}}=N, \quad w_{i}, w_{0}^{H_{x}} \geq 0
$$

by the same argument of the previous section. One can read off the surviving gauge group from the extended Dynkin diagram after removing of the corresponding circle for $\alpha_{i}$ or $\alpha^{H_{x}}$ if $w_{i} \neq 0$ or $w_{0}^{H_{x}} \neq 0$.

If there are more than one Wilson line, we can do this procedure recursively with every extended root $\alpha^{H_{x}}$ at the previous stage being taken care of by the same way as $\alpha^{0}$ is in the above illustration. As we took more Wilson lines, surviving gauge group has more factor groups with smaller rank, leading to many extended roots to be taken care of. It may become quite tedious but is trivial at the same time since we deal with small groups like $S U(n)$ for which all Coexter label is one.

\section{A. Further breaking of $S U(3) \times E_{6}$}

As an illustration of the method presented in the previous section. we will study the further breaking of $S U(3) \times E_{6}$ by one Wilson line. The subgroup $S U(3) \times E_{6}$ is obtained when the orbifold shift vector is given by $V=\gamma_{2} / 3$ for $Z_{3}$ orbifold, as can be seen from the TABLE 【. The $E_{6}$ has $\alpha^{3}, \cdots, \alpha^{8}$ as its simple roots and the highest weight is given by

$$
\theta^{E_{6}}=-\alpha^{E_{6}}=\alpha^{3}+2 \alpha^{4}+3 \alpha^{5}+2 \alpha^{6}+\alpha^{7}+2 \alpha^{8} .
$$

On the $S U(3)$ side, the simple roots consists of $\alpha^{1}$ and $\alpha^{0}$, the $E_{8}$ extended root, and the highest weight is given by

$$
\theta^{S U_{3}}=-\alpha^{S U_{3}}=\alpha^{0}+\alpha^{1}
$$

The extended Dynkin diagrams for $S U(3) \times E_{6}$ are shown in the FIG. 3 ,

1. Take $a=\frac{1}{3} \gamma_{4}$, or $w=\left[00010000 \mid w_{0} w_{0}^{S U_{3}} w_{0}^{E_{6}}\right]$. Since $\sum n_{i} w_{i}=5 \neq 0 \bmod 3, \alpha^{0}$ fails to pass the projection. $w_{0}$ is determined to be 1 with $w_{2}=-2$ from Eq.(21). Having $n_{0}^{S U_{3}}=1$ and $n_{4}^{E_{6}}=2$, the other coefficients are determined to be $w_{0}^{S U_{3}}=2$ and 

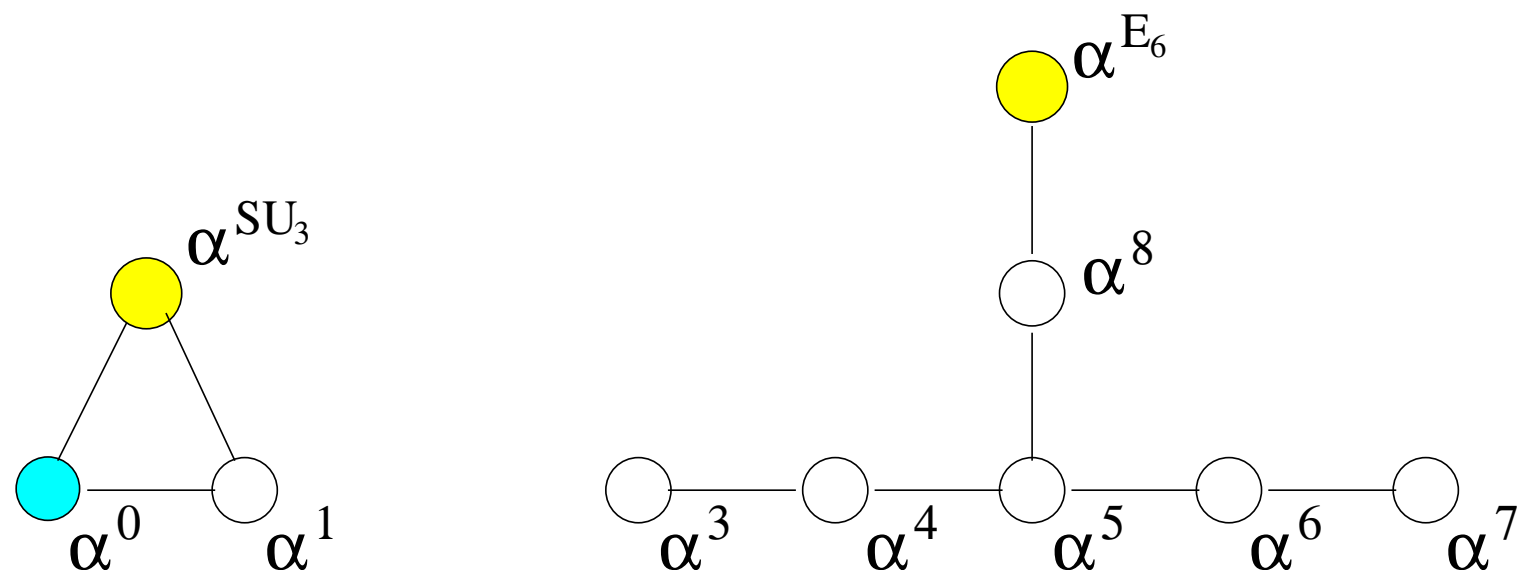

FIG. 3: Extended Dynkin diagrams for $\widehat{S U(3)}$ and $\widehat{E_{6}}$. New labels are defined for each subgroup. Compare these with FIG. 1.

$w_{0}^{E_{6}}=1$ from Eq.(19) and (20). The resulting group is $S U(2) \times S U(2) \times S U(5)$ and the final $w$ leading to this group is $w=[00010000 \mid 121]$. We leave $w_{2}$ to be zero for simplicity in this paper since it gives no information about the group structure.

2. Consider $a=\frac{1}{3} \gamma_{8}$. In this case $\alpha^{0}$ survives since $\sum n_{i} w_{i}=3$. Thus no simple root in $S U(3)$ group fail to pass the projection. We can take either $w_{0}=0$ or $w_{0}=3$, the result is the same, being $w_{0}^{S U_{3}}=3$ or $w_{0}^{S U_{3}}=0$, respectively. For $E_{6}$ part, $n_{8}^{E_{6}}=2$, thus we have $w_{0}^{E_{6}}=1$. As a result, we have $w=[00000001 \mid 031]$ and the resulting group is $S U(3) \times S U(6)$.

3. A more interesting case is $a=\frac{1}{3} \gamma_{5}$, or $w=[00001000 \mid 030]$. Both $\alpha^{0}$ and $\alpha^{E_{6}}$ survive. Therefore, a fairly large group of $S U(3)^{4}$ survives. By looking at the Dynkin diagram, one notes a symmetry by permutating three of $S U(3)$ 's. This has been used for an $S U(3)^{3}$ unification model 11$]$.

In this way, all possible other $w_{i}$ 's are examined and the resulting gauge groups are listed in TABLE II] Some comments are in order. The extended Dynkin diagram of $\widehat{E_{6}}$ shows the symmetry, namely triality, under permutations of $\left(\alpha^{3}, \alpha^{4}\right),\left(\alpha^{7}, \alpha^{6}\right)$ and $\left(\alpha^{E_{6}}, \alpha^{8}\right)$ pairs. Nonetheless, the breakings of $E_{6}$ by deleting the corresponding spots under this triality are not Weyl-equivalent since they have different $E_{8}$ characteristic like the Coexter labels $\left\{n_{i}\right\}$. As a result, it can be easily seen that some $w$ vectors resulting in the same gauge group are related by the triality symmetry. 


\begin{tabular}{|c|c|}
\hline$\left[w_{i} \mid w_{0} w_{0}^{S U_{3}} w_{0}^{E_{6}}\right]$ & group \\
\hline$[10000000 \mid 113]$ & $E_{6}$ \\
\hline$[00100000 \mid 212]$ & $S U(2) \times S O(10)$ \\
\hline$[00000010 \mid 122]$ & $S U(2) \times S O(10)$ \\
\hline$[00010000 \mid 121]$ & $S U(2)^{2} \times S U(5)$ \\
\hline$[00000100 \mid 211]$ & $S U(2)^{2} \times S U(5)$ \\
\hline$[00000001 \mid 031]$ & $S U(3) \times S U(6)$ \\
\hline [00001000|000] & $S U(3)^{4}$ \\
\hline$[10100000 \mid 022]$ & $S U(2) \times S O(10)$ \\
\hline$[10000010 \mid 202]$ & $S U(2) \times S O(10)$ \\
\hline$[10010000 \mid 201]$ & $S U(2)^{2} \times S U(5)$ \\
\hline$[10000100 \mid 021]$ & $S U(2)^{2} \times S U(5)$ \\
\hline$[10000001 \mid 111]$ & $S U(6)$ \\
\hline$[10001000 \mid 110]$ & $S U(3)^{3}$ \\
\hline$[00110000 \mid 030]$ & $S U(3) \times S U(6)$ \\
\hline$[00000110 \mid 030]$ & $S U(3) \times S U(6)$ \\
\hline$[00100100 \mid 120]$ & $S U(2)^{2} \times S U(5)$ \\
\hline$[00010010 \mid 210]$ & $S U(2)^{2} \times S U(5)$ \\
\hline$[00100001 \mid 210]$ & $S U(2)^{2} \times S U(5)$ \\
\hline$[00000011 \mid 120]$ & $S U(2)^{2} \times S U(5)$ \\
\hline$[00100010 \mid 031]$ & $S U(3) \times S O(8)$ \\
\hline$[10110000 \mid 110]$ & $S U(6)$ \\
\hline$[10000110 \mid 110]$ & $S U(6)$ \\
\hline$[10100100 \mid 200]$ & $S U(2)^{2} \times S U(5)$ \\
\hline [10010010|020] & $S U(2)^{2} \times S U(5)$ \\
\hline$[10100001 \mid 020]$ & $S U(2)^{2} \times S U(5)$ \\
\hline$[10000011 \mid 200]$ & $S U(2)^{2} \times S U(5)$ \\
\hline$[10100010 \mid 111]$ & $S O(8)$ \\
\hline
\end{tabular}

TABLE II: Possible further breakings of $E_{6} \times S U(3)$. $U(1)$ factors are implied to make the rank 8. Note that $\sum n_{I} w_{I}=0 \bmod 3, \sum n_{i}^{E_{6}} w_{i}=3, \sum n_{i}^{S U_{3}} w_{i}=0$. The shift vectors resulting in the same group are related by a symmetry. We do not list the cases with some $w_{i}$ 's of 2 , because they do not provide any new symmetry breaking patterns. 


\section{HETEROTIC STRING AND MODULAR INVARIANCE}

So far the procedure presented for finding an unbroken subgroup of $E_{8}$ by the Dynkin diagram has been general. In string theory, however, the consistency under the quantum corrections demands further conditions which is called the modular invariance conditions. In this case, the unbroken group by the shift vectors is more restricted. The search for the groups via shift vectors was the original motivation for introducing the orbifold compactification [2] in physics. In fact, these root systems naturally arise from the heterotic string [1]. Here, we employ the bosonic string description. A string state excited by an oscillator describes a Cartan generator in Eq. (2). The momentum and winding states around the compact dimension, which describe "charged bosons" under this Cartan generator describe roots in Eq. (11). The modular invariance condition restricts the relations among these states.

By orbifolding we identify the space (in the compact dimension coordinate $z_{m}=x_{2 m}+$ $\left.i x_{2 m+1}\right)$

$$
z_{m} \sim z_{m} e^{2 \pi i \phi_{m}}
$$

Similarly shift vector is defined on the group space

$$
z_{n} \sim z_{n} e^{2 \pi i v_{n}}
$$

By the definition of order, $N$ successive twist is identity operation, hence

$$
\sum N \phi_{m}=\sum N v_{n}=0 \quad(\bmod 2)
$$

where the modulo 2 condition results for the case of spinorial states, e.g. in the $E_{8} \times E_{8}^{\prime}$ theory.

Here, the modular invariance condition 2] for the orbifold restricts the form of the shift vector. Constraining the discussion on the abelian orbifold, i.e. when the orbifold action is

commutative 6] then the only necessity from the modular invariance condition [2] is that under $\tau \mapsto \tau+1$. In terms of the shift vectors $\phi$ and $V$, we have

$$
(N \phi)^{2}=(N V)^{2}, \quad(\bmod 2 N)
$$


where

$$
\begin{aligned}
(N V)^{2} & =\left(\sum s_{i} \gamma_{i}\right)^{2} \\
& =\sum_{i j} s_{i} A_{i j}^{-1} s_{j} \\
& =s \cdot A^{-1} s,
\end{aligned}
$$

where the dot product between two vectors in the Dynkin basis is understood. In this basis, we can easily read off the modular invariance condition from the inverse Cartan matrix $A^{-1}$. This is especially useful in implementing the procedure into the computer code. For a concrete form of the Cartan matrix, see [13].

\section{A. Wilson lines}

The presence of Wilson lines changes the modular invariance condition [2]. In addition to Eq. (26), we have the condition for each Wilson line shift $a_{i}$,

$$
(N \phi)^{2}=\left(N V+N m_{i} a_{i}\right)^{2} \quad(\bmod 2 N)
$$

Here, by the point group action as well as the lattice translation by $m_{i}$, the Wilson-lineshifted vector is made coincident to another shift vector. Since we have no preference on the choice of reference lattice axis, we can always set $m_{i}$ to be 1 . Therefore, one can show that this can be simply restated as,

$$
\begin{aligned}
& N V \cdot a_{i}=\text { integer } \\
& N a_{i} \cdot a_{j}=\text { integer }, \quad(i \neq j) .
\end{aligned}
$$

This can be written in the Dynkin basis as,

$$
w_{i} \cdot A^{-1} s=0 \quad(\bmod N)
$$

and

$$
w_{i} \cdot A^{-1} w_{j}=0 \quad(\bmod N, i \neq j)
$$

In the presence of more than one Wilson line, these conditions constrain the theory severely and reduce drastically the number of possibilities.

In the $E_{8} \times E_{8}^{\prime}$ heterotic string, we can independently consider two sectors independently. When we focus on the "visible" sector only, the modular invariance condition is loosened, 
because we can put the unwanted shift vector components into the hidden sector. For the modular invariance, the full rank-16 group must be considered for the condition (26). However, physics of the hidden sector is still important. When we consider twisted sectors, generally multiplets are hung on both sectors.

It is easily checked that these modular invariance conditions are not spoiled by the automorphisms of the group. As seen in TABLE [I] therefore the same groups that come from the equivalent breaking survive together if one survives.

\section{CLASSIFICATION SCHEME}

From the above construction, we can classify which group emerges when we orbifold. The general strategy for obtaining an orbifolded unbroken group is the following:

1. Determine the number of compact dimensions and the orbifold which determines the order $N$ of the shift.

2. Find all possible shift vector $v$ having the coefficient $s_{i}$ which satisfies Eq.(15) for given order $N$, for single gauge group $E_{8}$.

3. The allowed shift vector $V$ for $E_{8} \times E_{8}$ can be selected from the combination of two shift vectors for single $E_{8}$ by requiring the modular invariance condition (26).

4. The surviving gauge group for the chosen shift vector can be read off from the extended Dynkin diagram using the method explained in this paper.

5. Whenever we add Wilson line $a_{i}$, repeat the process from step 2, using Eq.(23) this time.

We note the merits of this procedure,

- Simple and intuitive.

- Complete classificiation: We can take into account all the symmetries from a given group.

- Easy to find the origin of a symmetry: We can see pictorially which symmetry comes from which group. From the example we presented earlier, the origin of $S U(3)^{3}$ from $E_{6}$ is easily seen. 
Since the above procedure is easily implementable in the computer program, we will present the classification tables of unbroken groups with shift vectors and Wilson lines, including the matter spectrum, in a separate publication[14].

In this paper, we set out the rules for finding out all the unbroken gauge groups with all the possible shift vectors and Wilson lines. The main point of the procedure we proposed is that we can exhaust the Weyl reflection symmetry by requiring the shift vectors and the Wilson lines to be the standard form for each subgroup, which is still simple enough to read off the surviving gauge group from the extended Dynkin diagram at the same time. Since we obtain all the allowed gauge groups with shift vectors and Wilson lines, the search for models with different sets of chiral matters within a given gauge group is much more simplified and tractable. It is even straight forward to identify the untwisted and twisted matter representations for every possible orbifold models, since we have done the classification of the Weyl-non-equivalent shift vectors and Wilson lines, if needed by the aid of the computer program [14].

\section{Acknowledgments}

We thank the Physikalisches Institut of the Universität Bonn for the hospitality extended to us during our visit when this work was completed. This work is supported in part by the KOSEF Sundo Grant(2002), the BK21 program of Ministry of Education, and Korea Research Foundation Grant No. KRF-PBRG-2002-070-C00022.

[1] D. J. Gross, J. A. Harvey, E. J. Martinec and R. Rohm, Phys. Rev. Lett. 54 (1985) 502; Nucl. Phys. B256 (1985) 253; Nucl. Phys. B267 (1986) 75.

[2] L. J. Dixon, J. A. Harvey, C. Vafa and E. Witten, Nucl. Phys. B261 (1985) 678; Nucl. Phys. B274 (1985) 285.

[3] L. E. Ibanez, H. P. Nilles and F. Quevedo, Phys. Lett. B187 (1987) 25.

[4] L. E. Ibanez, J. E. Kim, H. P. Nilles and F. Quevedo, Phys. Lett. B191 (1987) 282.

[5] C. A. Casas, E. K. Katehou, and C. Munoz, Nucl. Phys. B317 (1989) 171; A. Font, L. E. Ibanez, F. Quevedo and A. Sierra, Nucl. Phys. B331 (1990) 421; D. Bailin, A. Love and S. Thomas, Phys. Lett. B194 (1987) 385. 
[6] L. E. Ibanez, J. Mas, H. P. Nilles and F. Quevedo, Nucl. Phys. B301 (1988) 157.

[7] Y. Katsuki, Y. Kawamura, T. Kobayashi, N. Ohtsubo, Y. Ono and K. Tanioka, DPKU-8904 (1989).

[8] J. A. Casas, M. Mondragon, and C. Munoz, Phys. Lett. B209 (1988) 214; J. Giedt, Annlas Phys. 289 (2001) 251; Annals Phys. 297 (2002) 67.

[9] Y. Kawamura, Prog. Theor. Phys. 103 (2000) 613 hep-ph/9902423; Prog. Theor. Phys. 105 (2001) 999 hep-ph/0012125.

[10] K. S. Babu, S. M. Barr, and B. Kyae, Phys. Rev. D65 (2002) 115008 hep-ph/0202178; K. Hwang and J. E. Kim, Phys. Lett. B540 (2002) 289 hep-ph/0205093.

[11] J. E. Kim, hep-th/0301177.

[12] V. G. Kac and D. H Peterson, in Anomalies, Geometry, and Topology, p. 276-298, Proceedings of the 1985 Argonne/Chicago Conference; T. J. Hollowood and R. G. Myhill, Int. J. Mod. Phys. A3 (1988) 899; J. O. Conrad, Ph.D. Thesis, (Universität Bonn, 2001); Y. Katsuki, Y. Kawamura, T. Kobayashi, N. Ohtsubo, Y. Ono, and K. Tanioka, Nucl. Phys. B341 (1990) 611.

[13] H. Georgi, Lie algebras in particle physics, 2nd ed., Pergamon Press; R. Slansky, Phys. Rep. 79 (1981) 1.

[14] K.-S. Choi, K. Hwang, and J. E. Kim, to be published. 